\title{
Test Results for HINS Focusing Solenoids at Fermilab
}

\author{
M.A. Tartaglia, D.F. Orris, I. Terechkine, J.C. Tompkins
}

\begin{abstract}
A focusing lens $R \& D$ program is close to completion and industrial production of magnets has begun. Two types of magnets are being built for use in the room temperature RF section at the front end of a superconducting $\mathrm{H}$-minus linac of a High Intensity Neutrino Source. All of the magnets are designed as a solenoid with bucking coils to cancel the field in the vicinity of adjacent RF cavities, and one type incorporates steering dipole corrector coils. We present a summary of the predicted and measured quench and magnetic properties for both $R \& D$ and production device samples that have been tested at Fermilab.
\end{abstract}

Index Terms - linac, solenoid, focusing, magnet, quench, performance

\section{INTRODUCTION}

$\mathrm{T}$ HE development of superconducting solenoid lenses to focus intense $\mathrm{H}$-minus ion beams has been ongoing for several years at Fermilab as part of the high intensity neutrino source (HINS) R\&D program [1]. The basic lens design is constrained by the need to have a short magnet with strong central field of up to $7 \mathrm{~T}$, but with very low field at adjacent RF cavities a short distance from the magnet. The resulting design [2], [3] consists of a main solenoidal coil with narrow field-cancelling coils at the ends. All of the coils use coated NbTi superconductor with bare strand diameters ranging from $0.3 \mathrm{~mm}$ to $0.8 \mathrm{~mm}$, and copper fraction of about 0.5 .

Focusing lenses are needed in the low energy sections of the linac, and three separate designs are required to provide stronger focusing with increasing energy. Three solenoid designs are identified as $\mathrm{CH}$, SSR1, and SSR2, according to the type of accelerating RF cavities used in these sections (we use shortened device names here; see references for full official names). For efficient axially symmetric focusing, it is necessary to correct beam position errors with horizontal and vertical dipole steering coils in some of the magnets. Magnets without position correcting coils are labeled Type-1, and those with steering dipoles are designated Type-2. Fig. 1 shows the design of a Type- 2 solenoid cold mass. Type- 1 and Type- 2 main coil geometries differ slightly for $\mathrm{CH}$ solenoids. A new narrow design of the dipoles will allow both of the SS1 types to have the same main coil geometry [4].

Manuscript received 26 August 2008. Work supported by the U.S. Department of Energy under contract No. DE-AC02-07CH11359.

D. F. Orris, M. A. Tartaglia, I. Terechkine and J. C. Tompkins are with Fermi National Accelerator Laboratory, P.O. Box 500, Batavia, IL 60510 USA. Corresponding author M. A. Tartaglia; phone: 630-840-3890; e-mail: tartaglia@fnal.gov.

\section{Magnet Overview}

The solenoid development program began with a series of three test coils to validate the basic design and explore manufacturing and performance issues. Tests of those coils showed good agreement with predicted mechanical, quench and magnetic performance [5]. Further development of the winding techniques to achieve a consistently high packing factor, and incorporation of the bucking coils and steering correction dipole coils then began with the fabrication of two prototype $\mathrm{CH}$-section solenoids. One Type-1 prototype magnet, $\mathrm{CH} \_01$, and one Type-2 prototype, $\mathrm{CH} \_02$, were built and tested [3], [6], [7]. The former was then welded into a helium vessel and tested again, as CH_01-1, to check that after welding the quench behavior did not change.

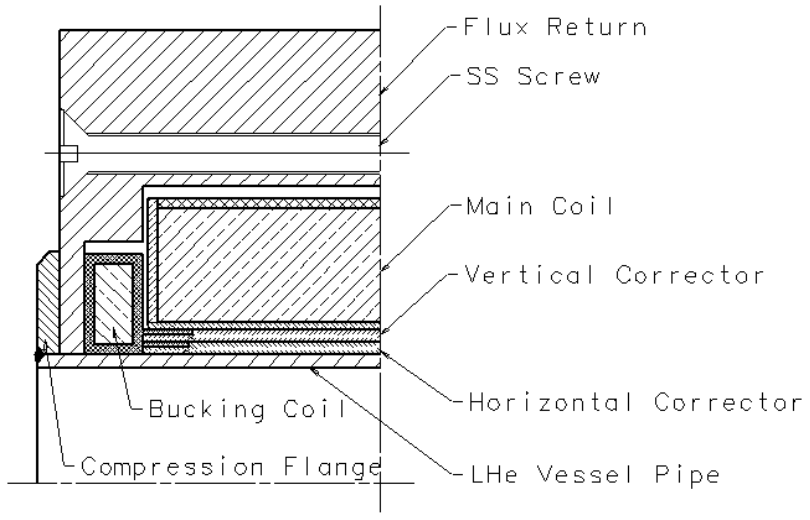

Fig. 1. Design of a Type-2 focusing solenoid.

Some design changes were deemed necessary to make the correction dipoles easier to manufacture and to increase their operating margin. The nesting of dipole coils required a change to the main solenoid coil dimensions, and some redesign of the bucking coil geometry to optimize their performance as well. Other changes to the design of both types was made: replacing the copper bobbins with G-10 to reduce cost and ground insulation concerns, and axial force adjustments to ensure no gaps in bucking coil support.

Two pre-production Type-2 solenoids, CH_03d and CH_04d, and two pre-production Type-1 solenoids, CH_05 and CH_06, were then built and tested ("d" was added in the names to easily identify solenoids with dipole coils) [8]-[11]. After a quench performance anomaly (discussed later), CH_03d was rebuilt with new bucking coils as CH_03d-1 and successfully re-tested [12].

Most of the coils in these pre-production magnets were fabricated using the same superconductor strand that was 
eventually shipped to the vendor selected to build 23 production solenoid magnets, Cryomagnetics, Inc. So far, two production Type-1 solenoids have been built and tested by the vendor, the first of which was also successfully re-tested after welding into the helium vessel. Re-testing at Fermilab of approximately one in four of the production magnets is planned as a quality assurance step prior to installation into production cryostats. CH_01-1 was mounted in a prototype cryostat and the assembly has been tested [13].

Development of solenoids for the superconducting RF cavity sections is also taking place. The first Type-1 prototype SSR1 solenoid has been built and tested [14], and a Type-2 prototype is now under construction. Design of the SSR2 solenoids, in which quench protection issues are a greater concern, is under way.

\section{QUENCH PERFORMANCE}

\section{A. Overview}

The magnets were tested in the Magnet Test Facility at Fermilab in the "test stand 3" vertical dewar, as described in [4]. However, because of the need to power multiple coils simultaneously, the top plate assembly was augmented to have three pairs of 500 A vapor cooled leads. The system of four "125 A" Lakeshore power supplies was split to deliver up to 275 A - the experimentally determined limit - to two separate circuits simultaneously (for dipole quench tests in the solenoid field). The quench protection system was reconfigured so that any detected quench (by whole coil voltage) would phase off all power supplies, but to use a dump switch and resistor only on the solenoid circuit. The splices were instrumented with voltage taps for determination of the quenching coil, as well as to allow protection of the superconducting leads.

Quench performance has been estimated for all of the tested magnets by determining the intersection of measured strand critical surface with the as-built load line for each coil. For Type-2 prototype solenoids which were built using production superconductor strand and with geometry very close to the nominal design, limiting quench is expected in the main coil at 233 A. For Type- $1 \mathrm{CH}$ solenoids, the predicted limit occurs in the main coil at $244 \mathrm{~A}$.

Main, bucking, and correction dipole coils generally are made from strands of different diameter. Critical current versus applied field measurements were made for samples from each lot of strand used to build a coil. Note that some temperature variation in the test dewar, from 4.2 to $4.4 \mathrm{~K}$, is possible from pressure being slightly over 1 atm; short sample curves (measured at $4.2 \mathrm{~K}$ ) are corrected using NbTi critical surface parametrization [15]. Load-lines were calculated from 2-D models in the Opera (Vector Fields) and COMSOL (Multiphysics) analysis programs, using the as-built geometry of each individual magnet assembly to determine the peak field versus current in each coil.

\section{B. Solenoid Training and Ramp Rate Dependence}

Quench performance was explored by training each magnet at low ramp rate (1 or $2 \mathrm{~A} / \mathrm{s}$ ) to reach a plateau. The HINS linac does not require fast ramping, so higher ramp rate behavior was not systematically investigated. The training plan for R\&D solenoids evolved somewhat through the program. There was interest to explore the behavior of individual coil types, as well as the bucked-coil magnet. For the prototypes CH_01 and CH_02, training was conducted on the bucking coils, then main coil alone, then bucked main coil. The predicted quench limit for bucking coils alone was $300 \mathrm{~A}$, well above the power supply limit; training of the separate coils did not eliminate training in the bucked-coil solenoid.

Subsequent pre-production magnet tests usually followed a simple program of training only in the bucked main coil configuration. The early test solenoids [5] trained very fast: they required four quenches or less to reach their maximum current. The prototype and pre-production solenoids generally trained more slowly. Two Type-1 production magnets, CH_T1_01 and CH_T1_02, have been tested so far by the vendor. Both have reached the required training level, $90 \%$ of the predicted quench limit, with one low current quench.

Table I summarizes quench training of all the bucked main coil magnets in this program: the predicted and maximum current reached, number of training ramps needed to reach or exceed the model-predicted Iq and $90 \%$ of Iq; currents shown in parentheses were limited by the power system, not magnet.

TABLE I. SOLENOID QUENCH SUMMARY

\begin{tabular}{l|c|c|c|c}
\hline \hline \multicolumn{1}{c|}{ Solenoid } & Iq model [A] & Iq max [A] & N (Iq) & N (0.9 Iq) \\
\hline CH_01 & 255 & 256 & 6 & 3 \\
CH_01-1 & 260 & 260 & 3 & 2 \\
CH_02 & 275 & $(260)$ & $(2)$ & 2 \\
CH_03d & 233 & 232 & 33 & 26 \\
CH_03d-1 & 233 & 240 & 7 & 5 \\
CH_04d & 233 & 235 & 24 & 12 \\
CH_05(wp) & 235 & 237 & 35 & 19 \\
CH_05 & 244 & $(242)$ & $(4)$ & 3 \\
CH_06 & 244 & 250 & 13 & 5 \\
CH_T1_01 & 244 & $(225)$ & $(2)$ & 2 \\
CH_T1_02 & 244 & $(225)$ & $(2)$ & 2 \\
SS1_01 & 220 & 225 & 33 & 15 \\
\hline \hline
\end{tabular}

The freedom to connect the individual coils externally led to a particularly interesting history for solenoid $\mathrm{CH}_{-}$05: one bucking coil was inadvertently operated with the wrong polarity (wp), it's field parallel to the main coil. This was not discovered until magnetic measurements later revealed an asymmetric profile, after training had ended with the main coil quenching at $237 \mathrm{~A}$, well below the expected $245 \mathrm{~A}$. Modeling of this configuration predicts a quench current of $235 \mathrm{~A}$. After a thermal cycle, it was re-trained with correct field polarities.

One important lesson learned during the training of $\mathrm{CH} \_05$ was to wait sufficient time between quenches; specifically, bucking coil quenches dissipate much of the main coil energy and experience the highest temperature rise [3]. A delay of ten minutes after a bucking coil quench was not sufficient, and would reproducibly result in another quench in the same bucking coil, at lower current (and contribute to slow training). This hypothesis is yet to be confirmed by modeling 
to predict the heat diffusion time constant.

This effect became relevant as a plausible explanation for the puzzling behavior of $\mathrm{CH} \_03 \mathrm{~d}$, which had two low current bucking coil quenches after training. The test was then ended, the magnet was disassembled, the suspect bucking coil was "ring" tested and dissected: it was deemed "perfect" with no evidence of shorts, winding or impregnation problems.

The CH and SS1 solenoids should be capable of surviving a quench without using an energy extraction resistor. This was tested in CH_01, CH_04d, CH_T1_01, CH_T1_02, and SS1_01, which were quenched several times without a dump resistor to demonstrate survival without degradation. The vendor will test all production $\mathrm{CH}$ solenoids in this manner.

Two of the R\&D magnets and both production magnets were put through a thermal cycle: all returned to their trained quench current level without lower current quenches.

Ramp rate dependence was measured for magnet $\mathrm{CH}_{-}$06 after training, and for CH_01-1 in the prototype cryostat. As shown in Fig. 2, there is little ramp rate variation up to $6 \mathrm{~A} / \mathrm{s}$, and the plateau quench current fluctuates by a few Amperes.

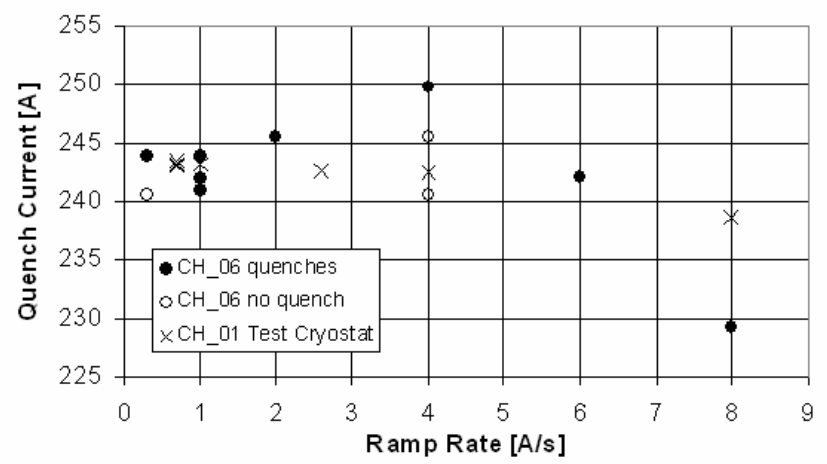

Fig. 2. Ramp rate dependence for $\mathrm{CH} \_06$ and $\mathrm{CH} \_01-1$. "No quench” points were currents reached, but limited by power supply ramp control problems.

\section{Steering Correction Dipoles}

Each of the correction dipole coils was tested after the solenoid had been trained to the operating current level. With the solenoid powered at $200 \mathrm{~A}$, the two dipoles were ramped in series to the power supply limit of 275 A without a quench in either dipole or solenoid. In the prototype $\mathrm{CH}_{-} \mathrm{02d}$, a current of 250 A was needed to reach the integrated strength requirement of $0.25 \mathrm{~T}-\mathrm{cm}$, while the re-designed coils in preproduction magnets $\mathrm{CH} \_03 \mathrm{~d}$ and $\mathrm{CH} \_04 \mathrm{~d}$ met the dipole strength requirement at $125 \mathrm{~A}$, and thus have very good operating margin.

\section{MAgnetic Performance}

\section{A. Overview}

A number of magnetic performance characteristics are important for the focusing lenses. In addition to the dipole strength mentioned above, the relevant $\mathrm{CH}$-solenoid field integral requirement is $\int \mathrm{B}^{2} \mathrm{dz}>1.8 \mathrm{~T}^{2}-\mathrm{m}$ along the (z) axis (>3.0 $\mathrm{T}^{2}-\mathrm{m}$ for SS1-solenoid). The solenoid is designed to have significant margin, and it is important to measure how well the devices match the predicted peak strength and axial profile. Equally important is how effective are the bucking coils and iron yoke at quickly reducing the field at the ends, so details of the fringe field region have been systematically studied. Field uniformity, especially for the dipoles, is also important for beam emittance preservation.

A description of the apparatus for measuring current and magnetic strength is given in [3]. The Group3 probe, limited to $3 \mathrm{~T}$ measurements along one axis, was replaced during this program by a 3-axis $10 \mathrm{~T}$ Hall probe (Senis/GMW) digitized using a Keithley 2700 multiplexing DMM. Simultaneous determination of the axial and transverse (or radial and azimuthal) field components were made at, or above the nominal operating current, both on and off the axis. For the SS1 prototype solenoid, with an aperture too small for the available warm bore, a cryogenic Hall probe (HSP-A) and Gauss-meter (GM-700) from Cryomagnetics, Inc. were used.

\section{B. Peak and Fringe Field Strength}

Profiles of the axial magnetic strength were measured for all magnets, operated over a range of currents, up to 230A; agreement with the as-built model predicted shape is excellent in all cases. The transfer function variation, shown in Fig. 3, is small - except for the first two prototypes, the measured values are within $2.5 \%$ of the model. The $\mathrm{CH}$ lens field integral is met at $182 \mathrm{~A}$ and $171 \mathrm{~A}$ for the Type- 1 and Type- 2 solenoids, respectively, which provides a quench current margin of about $35 \%$ for both types.

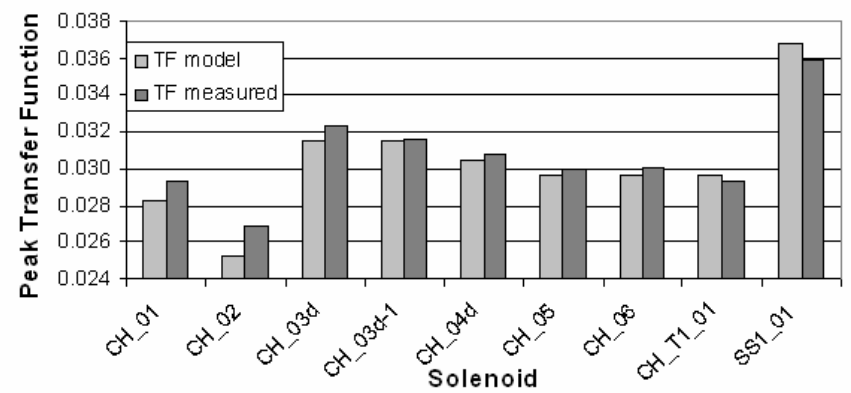

Fig. 3. Predicted and measured transfer functions (B/I) at the solenoid center.

The axial profiles are shown in Figures 4 and 5 for Type- 1 and Type-2 solenoids, respectively. Fig. 4 shows the model prediction for both types to illustrate the result of different coil geometries. The fringe fields, shown as inserts, are as good or better than expected. Both the peak and fringe fields have a dependence on current above $100 \mathrm{~A}$, due to iron saturation. The effect is greater off-axis, where the peak field falls off about $5 \%$ at $12 \mathrm{~mm}$ radius for the Type- 2 solenoid. The fringe field is more seriously affected, as shown in Fig. 6 for Type-1 solenoid CH_06 measured on-axis, and Type-2 solenoid CH_03d-1 measured at $12 \mathrm{~mm}$ off-axis in Fig. 7.

\section{Dipole Field Uniformity}

Correction dipole field uniformity was measured with a 3D Hall probe angle scan at high current, and confirmed the predicted $25 \%$ variation in the prototype $\mathrm{CH} \_03 d$ dipole field at $12 \mathrm{~mm}$ radius. The single-layer coil design was improved by adjusting winding angles: a harmonic coil probe measurement at room temperature with low current $(0.5 \mathrm{~A})$ shows $5 \%$ variation with angle. The optimized SS1 design 
predicts uniformity $10 \%$ at the beam tube radius, quite close to the coil: warm magnetic measurements have yet to be completed on the prototype SS1 coils to test predictions.

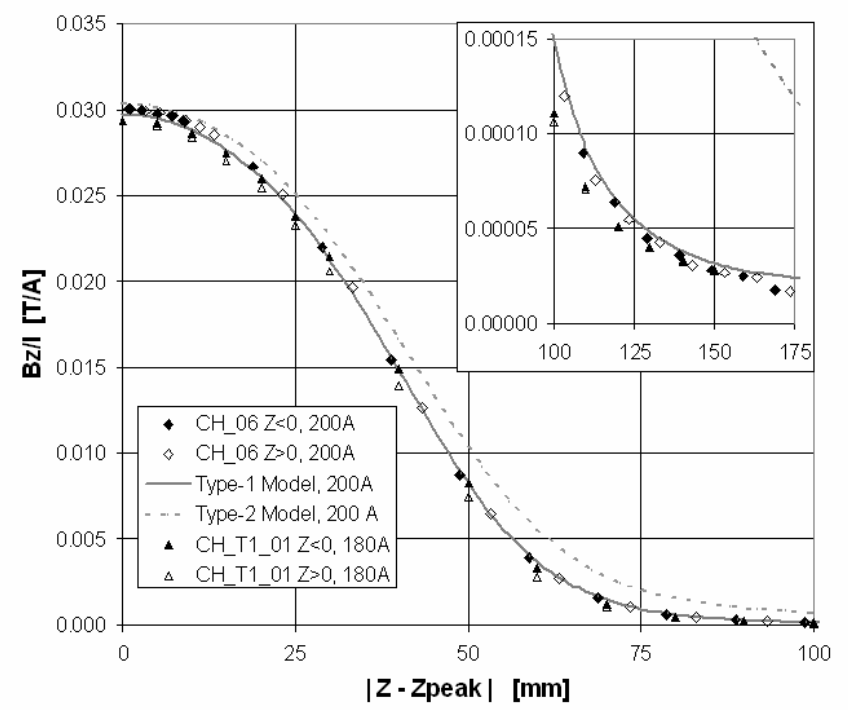

Fig. 4. Axial transfer function profiles for $\mathrm{CH} \_06$ (200 A) and CH_T1_01 (180A) compared to Opera model (200A) for Type-1 and Type-2 lenses, with fringe field detail shown in insert.

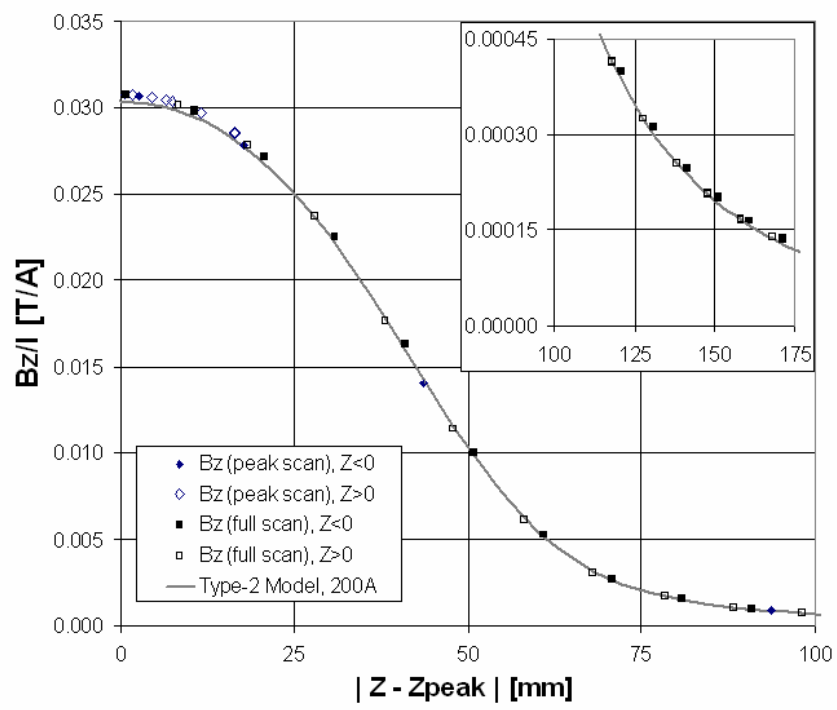

Fig. 5. CH_04d axial transfer function profile at 200 A compared to the Opera model (200A) for Type-2 as-built geometry; insert shows fringe field.

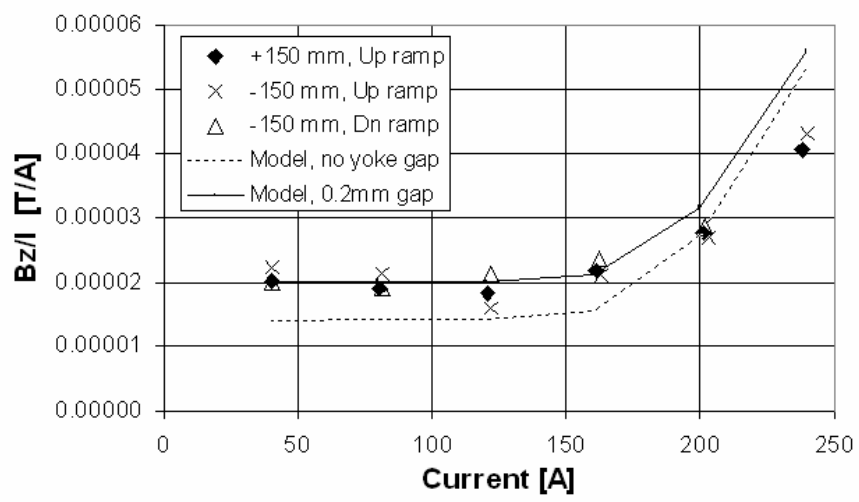

Fig. 6. Model and CH_06 axial transfer function profile versus current.

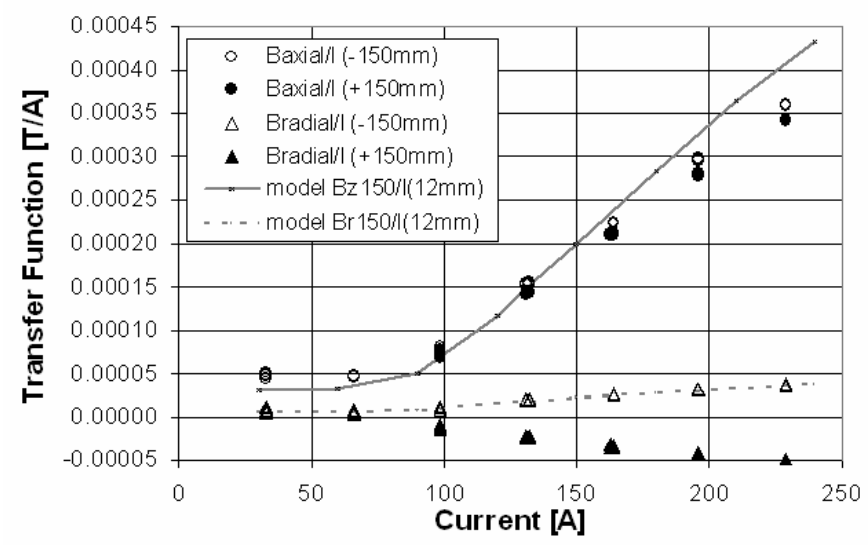

Fig. 7. Model and CH_03d axial transfer function profiles versus current.

\section{CONCLUSION}

The development of focusing solenoid lenses for the HINS $R \& D$ program is mature, and has evolved from the prototype stage to a production activity for the $\mathrm{CH}$ section of the beam line. Test results show good agreement with model expectations for quench and magnetic performance for all of the models tested. Prototype fabrication and testing is in progress for the SS1 section, and SS2 design work continues.

\section{REFERENCES}

[1] G.Apollinari, et al., "HINS Linac Front End Focusing System R\&D," Proceedings this conference.

[2] G. Davis, V.V. Kashikhin, T. Page, I. Terechkine, J. Tompkins, T. Wokas, "Designing Focusing Solenoids for Superconducting RF Accelerators," IEEE Trans. Appl. Supercon.., Vol. 17, pp.1221-1224, June 2006

[3] I. Terechkine, V.V. Kashikhin, T. Page, M. Tartaglia, J. Tompkins, "Focusing Solenoid for the Front End of a Linear RF Accelerator," 2007 Particle Accelerator Conference.

[4] G. Davis, M. Tartaglia, I. Terechkine, J. Tompkins, T. Wokas, "HINS Linac SS-1 Section Prototype Focusing Solenoid Design,” TD-08-010, FNAL, March 2008.

[5] R. Carcagno, et al., "Superconducting Solenoid Magnet Test Results," IEEE Trans. Appl. Supercond.., Vol. 17, pp.1213-1216, June 2006.

[6] C. Hess, et al., "Focusing Solenoid HINS_CH_SOL_01 Fabrication Notes and Test Results,” TD-07-006, FNAL, May 2007.

[7] C. Hess, et al., "Focusing Solenoid HINS_CH_SOL_02 Fabrication Notes and Test Results,” TD-07-008, FNAL, May 2007.

[8] E. Barzi, et al., "Expected Performance and Test Results of the First PreProduction Solenoid (Type-2, with correctors): HINS_CH_SOL_03d," TD-07-021, FNAL, August 2007.

[9] E. Barzi, et al., "HINS_CH_SOL_04d Expected Performance and Test Results,” TD-07-027, FNAL, October 2007

[10] E. Barzi, et al., "HINS_CH_SOL_05 Fabrication Summary and Test Results,” TD-07-031, FNAL, December 2007.

[11] G. Davis, et al., "HINS_CH_SOL_06 Fabrication Summary and Test Results,” TD-08-004, FNAL, January 2008.

[12] G. Davis, et al., "HINS_CH_SOL_03d-1 Fabrication Summary and Test Results,” TD-08-002, FNAL, January 2008.

[13] T.M. Page, et al., "HINS Superconducting Lens and Cryostat Performance," Proceedings this conference.

[14] G. Davis, et al., "HINS_SS1_SOL_01 Fabrication Summary and Test Results,” TD-08-012, FNAL, April 2008.

[15] L. Bottura, A practical fit for the critical surface of NbTi,” IEEE Trans. Appl. Supercond., Vol. 10, March 2000 